\title{
Primary structure and functional expression of a high voltage activated calcium channel from rabbit lung
}

\author{
Martin Biel ${ }^{1}$, Peter Ruth ${ }^{1}$, Eva Bosse' ${ }^{1}$, Roger Hullin¹, Walter Stühmer ${ }^{2}$, Veit Flockerzi ${ }^{1}$, Franz Hofmann ${ }^{1}$ \\ ${ }^{1}$ Medizinische Biochemie, Universität des Saurlandes, D-6650 Homburg/Saar, FRG and ${ }^{2}$ Max Planck-Institut für Biophysikalische
} Chemie, D-3400 Göttingen, FRG

Received 28 June 1990

The complete amino acid sequence of the receptor for organic calcium channel blockers (CaCB) from rabbit lung has been deduced by cloning and sequence analysis of the cDNA. Synthetic RNA derived from this cDNA induces the formation of a functional CaCB-sensitive high voltage activated calcium channel in Xenopus oocytes.

$\mathrm{Ca}^{2+}$ channel; Smooth muscle; Primary structure; Expression

\section{INTRODUCTION}

Voltage activated calcium channels are membrane spanning proteins that allow the controlled entry of $\mathrm{Ca}^{2+}$ ions into the cytoplasm of cells. This class of channels comprises a group of very similar yet distinct proteins or protein complexes that differ in kinetics, voltage dependence and their relative sensitivity to organic calcium channel blockers [1,2]. The principal subunit of a high voltage activated (L-type) calcium channel is the CaCB-receptor or $\alpha_{1}$ subunit [3-11]. So far, only the rabbit skeletal $[12,13]$ and cardiac muscle CaCB-receptors [14] have been cloned. Pharmacologically, the primary target for $\mathrm{CaCBs}$ is smooth muscle [15]. Assuming that airway smooth muscle cells are abundant in lung we have now isolated complementary DNAs for the CaCB-receptor from rabbit lung on the basis of sequence homology with its skeletal muscle counterpart.

\section{MATERIALS AND METHODS}

\subsection{Generation and screening of rabbit lung cDNA libraries}

A randomly primed cDNA library was constructed in pUC 9 using poly(A) + RNA prepared from adult rabbit lung. Screening with a rabbit skeletal muscle CaCB-receptor cDNA probe, that is EcoRI (nt 1007)-SacI(nt 5315) fragment from pCCH102 [12] gave 8 positive clones including pCaKL5 (nt 3957 to 5248) and pCaKL6 (nt 2452 to 4193 (Fig. 1). The PstI (nt 4899)-PstI(nt 5030) fragment from clone pCaKLS was used for screening of an oligo(dT)library and six positive clones including pCaKL34 (4792 to 6830 and a stretch of $10 \mathrm{dA}$ residues), pCaKl35 (4830 to 6826) and pCaKl25 (nt 4847 to 6829) were obtained. Elongation of a synthetic primer complementary to nt 2756 to 2773 and screening of the resulting clones with the PvuII (nt 2506)-

Correspondence address: V. Flockerzi, Medizinische Biochemie, Universität des Saarlandes, D-6650 Homburg/Saar, FRG
$A f 7 I(n t 2674)$ fragment yielded 4 positive clones including pCaKL 14 (nt 2769 to nt-544). Sequencing of the cDNA was performed on both strands. In addition pCaKL35 and pCaKL25 were sequenced. One nucleotide difference occurred among the individual clones: at nt $5422, \mathrm{G}$ (pCaKL34 and pCaKL35) or $\mathrm{T}$ (pCaKl25). The difference results in a substitution of Gly to Cys. Cloning procedures, if not stated otherwise, were carried out as described [16].

\subsection{Construction of recombinant plasmid for cDNA expression}

The recombinant plasmid pSCaL carrying the entire protein-coding sequence of the rabbit lung CaCB-receptor CDNA was constructed as follows. The $2.1 \mathrm{~kb}$ HindIII-BgIII fragment from pCaKL34, the 0.7 $\mathrm{kb} B g / \mathrm{II}-S a c I I$ fragment from pCaKL5, the $1.4 \mathrm{~kb}$ SacII-AfIII fragment from pCaK16 and the $3.0 \mathrm{~kb} A f l I I-X m a l$ fragment from pCaKL14 were subcloned in the $2.9 \mathrm{~kb}$ HindIII-Xmal fragment of pBluescript KS to yield pBCal. The $0.3 \mathrm{~kb} \mathrm{Bg}$ II-HindIII fragment (carrying a poly (dA) poly(dT) tract from pSPCA1 [14] was blunted by Klenow fragment, cleaved by $B a m H I$ and ligated with the $6.9 \mathrm{~kb}$ $B a m \mathrm{HI}-\mathrm{HpaI}$ fragment from pBCaL and the BamHI-cleaved pSP72 (Promega) to yield pSCaL.

\subsection{Expression of calcium channels in Xenopus oocytes}

The mRNA specific for the lung CaCB-receptor was synthesized in vitro using Asp718-cleaved pSCaL as template. Xenopus laevis oocytes were injected with the CaCB-receptor specific mRNA $(0.4$ $\mu \mathrm{g} / \mu \mathrm{l})$; the average volume injected was $50 \mathrm{nl}$. The injected oocytes were incubated as described [17] and the follicular cell layer was removed from oocytes before electrophysiological measurements. Whole cell currents were recorded at room temperature in (mM) 40 $\mathrm{Ba}^{2+}, 50 \mathrm{Na}^{+}, 2 \mathrm{~K}^{+}, 5 \mathrm{HEPES}$ ( $\mathrm{pH} 7.4$ with methanesulfonic acid). Current records were sampled at $0.5 \mathrm{~ms}$ intervals after low-pass filtering at $500 \mathrm{~Hz}$.

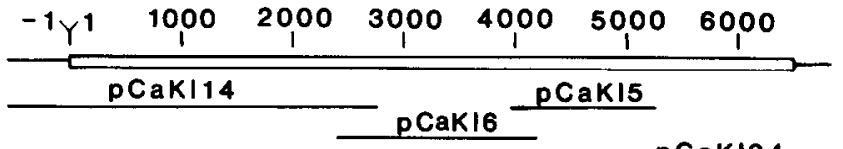

pCaK134

Fig. 1. Cloning strategy for the CaCB-receptor. The protein-coding region is indicated by a closed box. The extent of cDNA inserts of the individual clones used for sequence analysis are shown by thick lines. 


\section{RESULTS AND DISCUSSION}

Fig. 2 shows the 7374-nucleotide (nt) sequence obtained and the deduced primary structure. The translation initiation site was assigned to the first ATG triplet that appears downstream of nonsense codons found in frame. The lung CaCB-receptor is composed of 2166 amino acids having a relative molecular mass (Mr) of 242,516 . The amino acid sequence shows an overall homology of $65 \%$ to the skeletal muscle CaCB-receptor and differs from the rabbit heart receptor at four sites (Fig. 3): the $5^{\prime}$ end of the lung nucleotide sequence including the predicted amino terminus of the receptor (nucleotides -544 to +47 ) (site a), which is completely different from the respective sequence of the heart $\mathrm{CaCB}$ receptor; the nucleotide sequences encoding the predicted transmembrane segments IS6 (nucleotides 1114 to 1212) (site b) and IVS3 (nucleotides 3904 to 3987 ) (site d) which are 65\% (IS6) and 57\% (IVS3) identical at the nucleotide sequence level and $70 \%$ (IS6) and $54 \%$ (IVS3) identical at the amino acid sequence level, respectively; an insert of $75 \mathrm{nt}$ (nucleotide 1391 to 1465) (site c) which is not present in the cardiac receptor sequence. The remaining sequence of the lung receptor is identical to the cardiac receptor [14] including the number and localization of potential $\mathrm{N}$-glycosylation and cAMP-dependent phosphorylation sites.

Messenger RNA was synthesized by in vitro transcription of the cloned lung $\mathrm{cDNA}$ and injected into Xenopus laevis oocytes. To resolve the calcium current, external calcium was replaced by $\mathrm{Ba}^{2+}(40 \mathrm{mM})$ and external $\mathrm{Cl}^{-}$by methanesulphonate, as described

5 ....- CAGMGCTGCTCCCTGCCAGMCGGCGCGCGGCGCGGCGCGGCCCGGAGCGTGGCGGCTCCTCCTGCCTCCTCGCCTCAGGAGTTGGCGGCTC -45

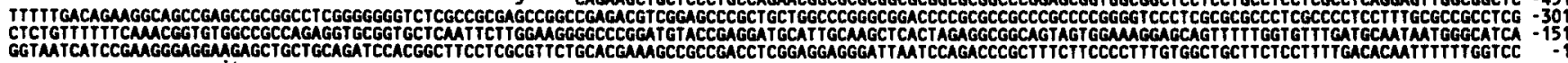

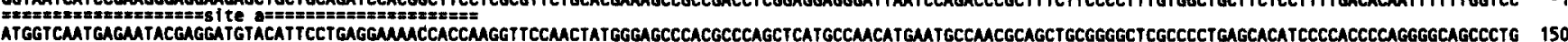
A

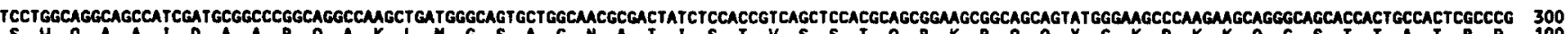
S O A A I D A A R O A K L H G S A G N A T I S T V S S T O R K R Q Q Y G K P K K Q G S T T A T R P I00

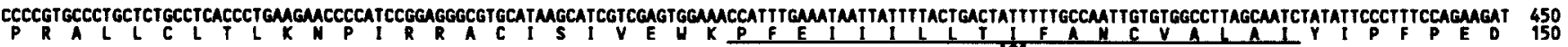

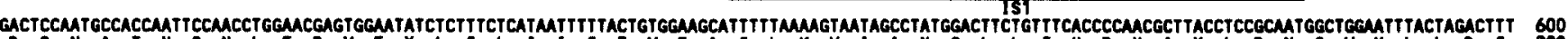

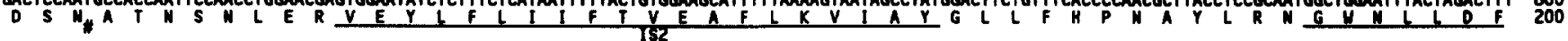

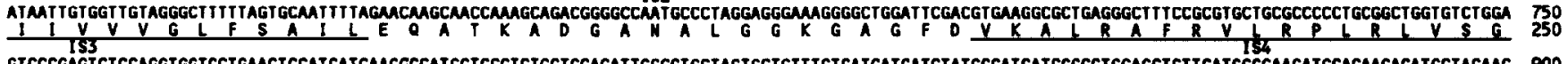

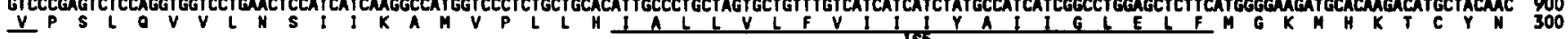

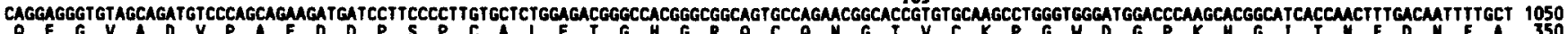
Q E G V A D V P A E D D P S P C A L E T G H G R O C A N G V V C

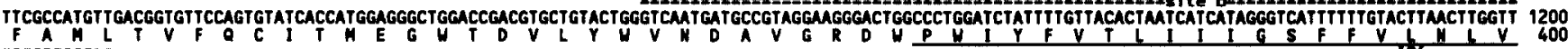

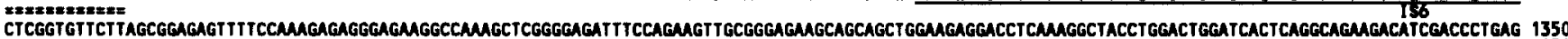

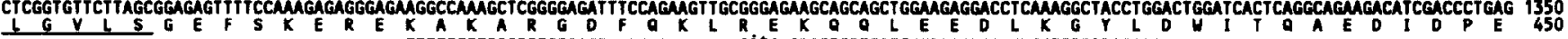

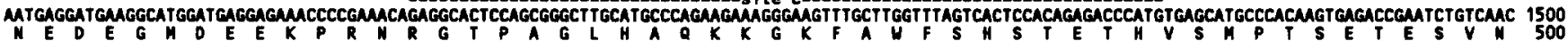

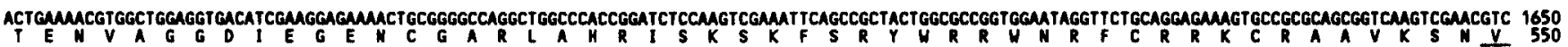

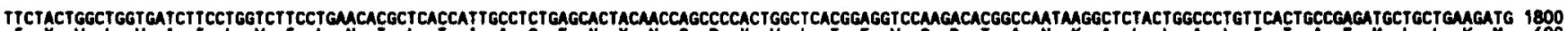

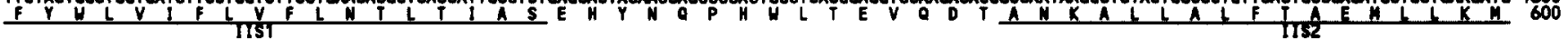
TACAGCCTGGGCCTGCAGGCCTATTTCGTGTCCCTCTTCMCCGCTTCGACTGCTTCATTGTGTGCGGGGCATCCTGGAGACCATCCTGGTGGAGACCMGGTCATGTCCCCCCTGGGCATCTCTGTOCTGACATECGTGCGGCTCCTG 1950

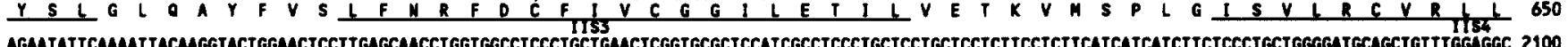

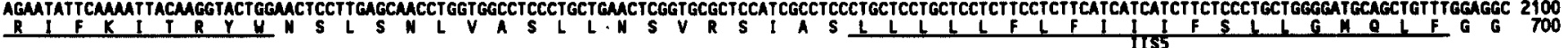

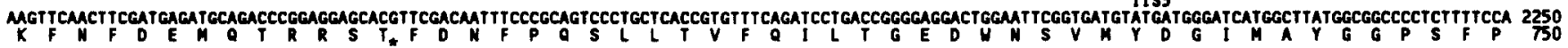

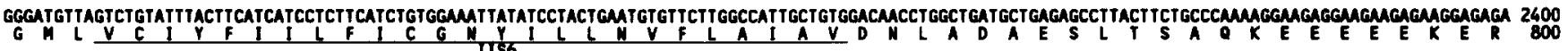

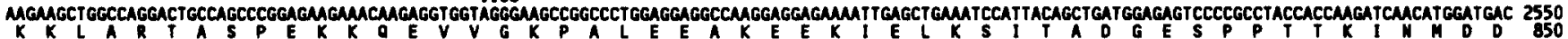

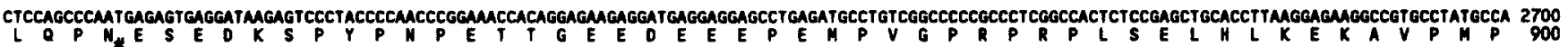

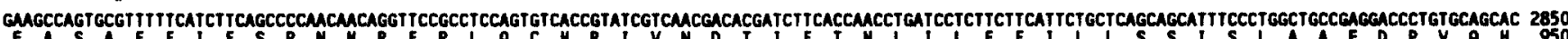

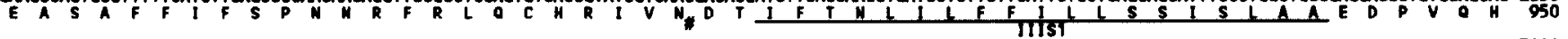

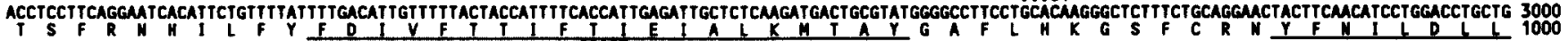

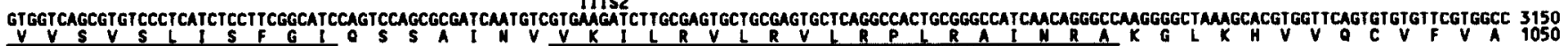

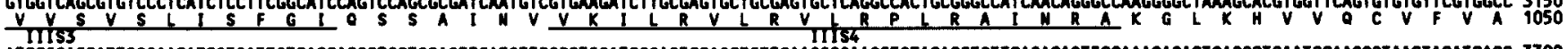

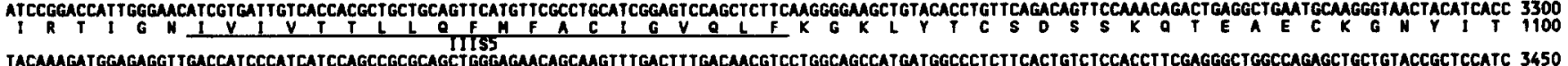

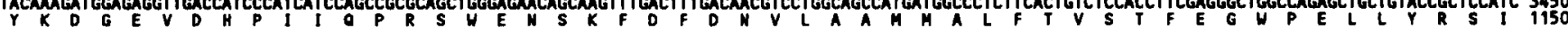

(continued)

Fig. 2. Nucleotide and deduced amino acid sequence of the CaCB-receptor. The predicted transmembrane segments $\mathrm{S} 1$ to $\mathrm{S6}$ in each of repeats I to IV of the CaCB-receptor are shown. The amino acid residues encoded by nucleotide sequences of sites a-d, the predicted phosphorylation sites for cAMP-dependent protein kinase (*) and potential $\mathrm{N}$-glycosylation sites (\#) are indicated. 


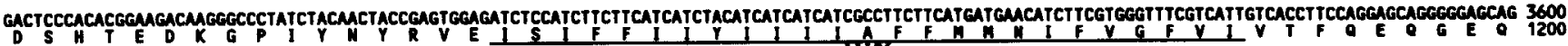
GAGTACMGMCTGTGAGCTGGACMGMCCAGCGGCAGTGCGTGGMTATECCCTCMGGCCCGGCCCCTGCGGGGTACATCCCCMGMCCAGCACCAGTACAMGTGTGGTACGTEGTCMACTCCACCTACTTTGAGTACCTGATG 3750

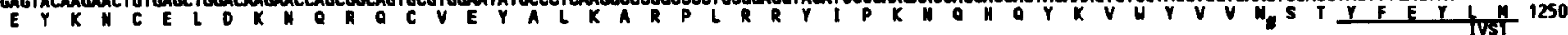

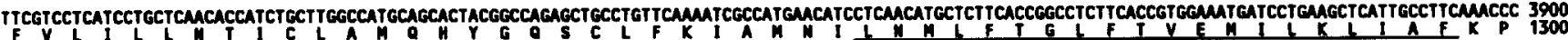

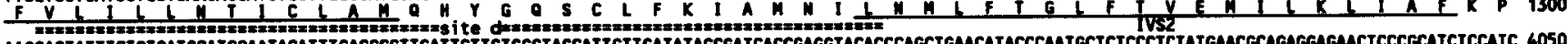

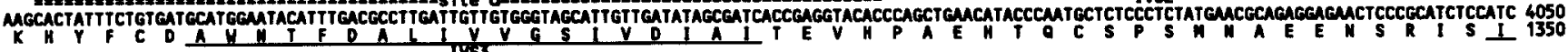

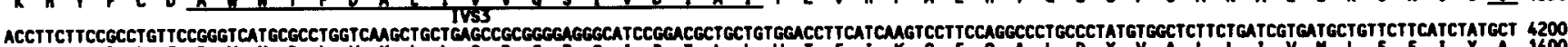

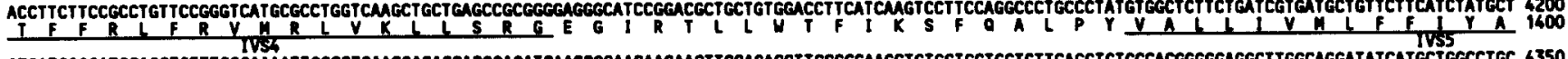

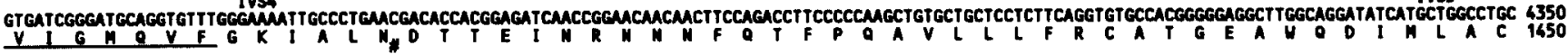
ATGCCAGGCMGMGTGTGCCCCAGAGTCTCACCCCCACMCAGCACAGMGGGGAGACCCCCTGCGGCAGCAGCTTCECCGTCTTCTACTTCATCAGCTTCTACATGCTTTGTGCCTTCCTGATCATCMTCTCTTTGTACCTGTCATC 4500 M P G K K C A P E S E P H N S T E G E T P C G S S F A V F Y F I S F Y H L C A F L I I I L F V V I I500

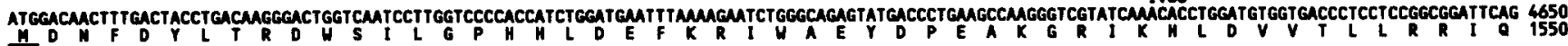

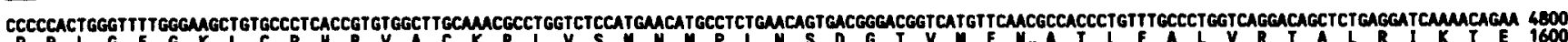
c

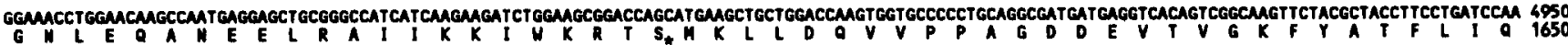

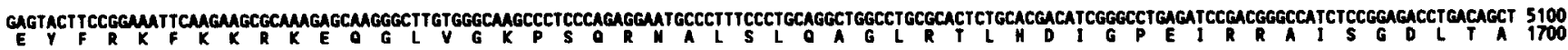

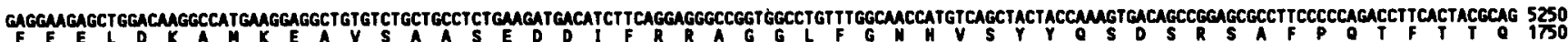

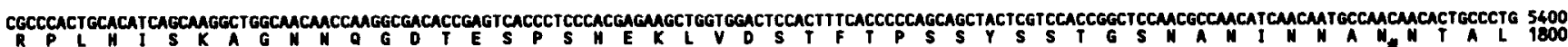

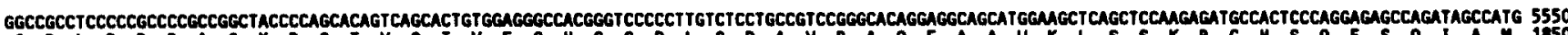

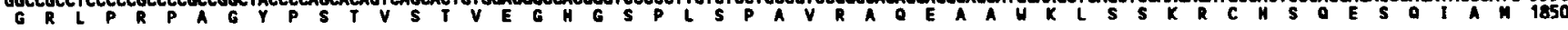

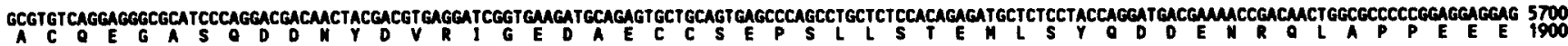

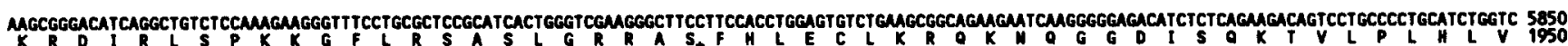

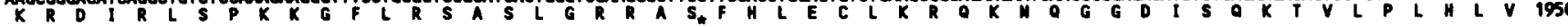

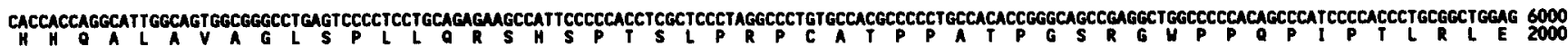

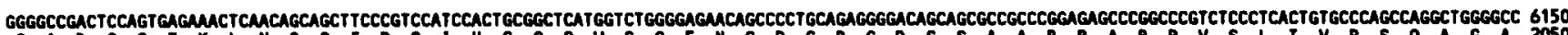

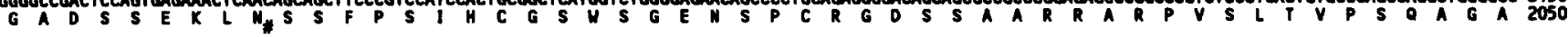

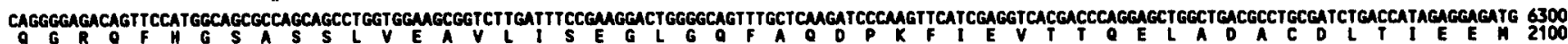

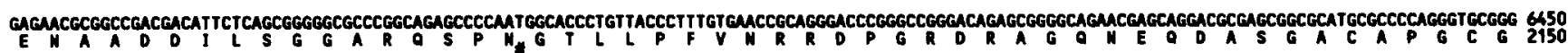

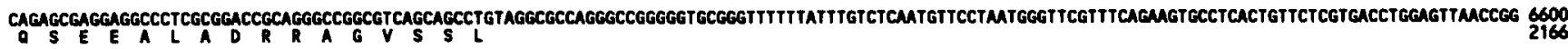

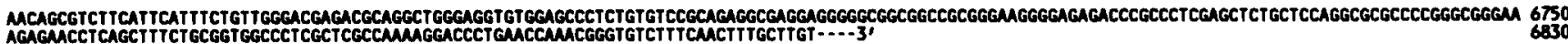

Fig. 2 (continued)

previously $[14,18]$. In this solution a sustained inward current was evoked by depolarizing pulses (Fig. 4); no such current was detected in non-injected oocytes. The inward current was insensitive to tetrodotoxin $(30 \mu \mathrm{M})$ or to the replacement of both $\mathrm{Na}^{+}$and $\mathrm{K}^{+}$with $\mathrm{Ba}^{2+}$ (final concentration, $100 \mathrm{mM}$ ) but was completely blocked by addition of $\mathrm{Cd}^{2+}(0.2 \mathrm{mM})$. The peak inward current was increased 2-fold by $5 \mu$ M BAY K 8644 whereas it was decreased to $20 \%$ of the control value by $1 \mu \mathrm{M}$ nifedipine and was virtually abolished by $10 \mu \mathrm{M}$ nifedipine. These results indicate that the inward cur-

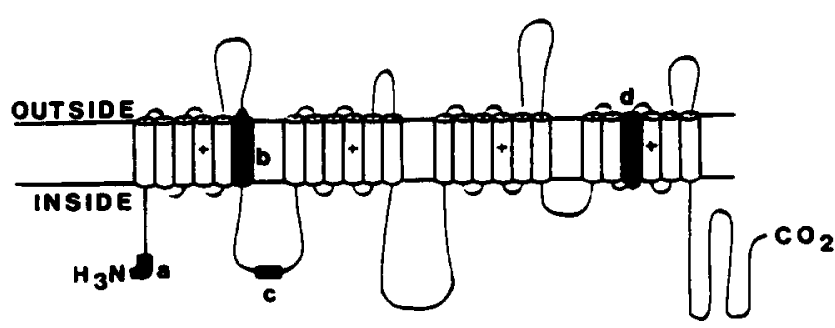

Fig. 3. Transmembrane folding model of the $\mathrm{CaCB}-$ receptor and approximate localization of sites $\mathrm{a}-\mathrm{d}$. rent is a barium current through a CaCB-sensitive calcium channel. Coinjection of mRNAs specific for the lung $\mathrm{CaCB}$ receptor and the rabbit skeletal muscle $\alpha_{2}$ polypeptide enhances the $\mathrm{Ba}^{2+}$ current without affecting channel kinetics. A similar $\alpha_{2}$ dependent increase in $\mathrm{I}_{\mathrm{Ba}}$ has been observed with the cardiac $\mathrm{CaCB}$ receptor [14]. These results show that the lung CaCBreceptor alone is sufficient to induce a $\mathrm{CaCB}$-sensitive calcium channel. It is not known whether or not the Xenopus oocyte expresses other proteins which are necessary for the expression of the functional channel. The CaCB-receptor clones from rabbit lung and heart are identical in the coding and noncoding nucleotide sequence except for the 4 sites described (see Fig. 3). This finding suggests that they arise by differential splicing of the same primary transcript. Preliminary results suggest that the CaCB-receptor cloned from rabbit lung is expressed in airway and vascular smooth muscle cells. In vivo L-type calcium channels from cardiac and smooth muscle have similar electrophysiological properties $[1,2]$. The primary sequences of the cardiac and the lung CaCB-receptor contain identical sites that could be phosphorylated by cAMP-dependent protein 


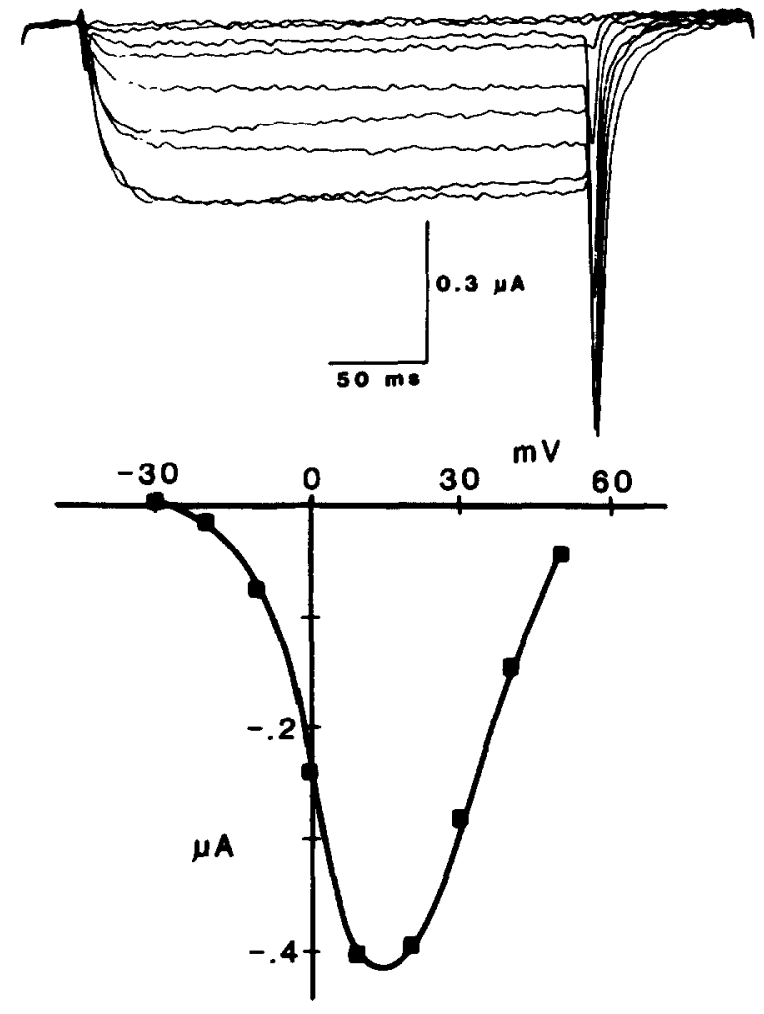

Fig. 4. (A) Current responses ( $\left.I_{B a}\right)$ in Xenopus oocytes injected with mRNA specific for the lung $\mathrm{CaCB}$-receptor. The depolarizations ranged from -30 to $50 \mathrm{mV}$ in $10 \mathrm{mV}$ steps from a holding potential of $-80 \mathrm{mV}$. (B) Current-voltage relation corresponding to the data shown in A.

kinase in vivo. However, the biochemical modulation of both channels appears to be different. cAMPdependent phosphorylation increases the cardiac calcium current [19] whereas it has little or no effect on the smooth muscle current [20]. Therefore stimulation of the calcium current might be not due to phosphorylation of the CaCB-receptor itself. Further work involving the stable expression of the CaCB-receptor in cells exhibiting appropiate signal transduction pathways will be required to test this hypothesis.
Acknowledgements: We thank T. Tanabe and S. Numa for kindly providing pSPCA1, R. Nagel for expert technical assistance and H. Siepmann for the graphical work. Supported by Deutsche Forschungsgemeinschaft, Thyssen and Fond der Chemischen Industrie.

\section{REFERENCES}

[1] Bean, B.P. (1989) Annu. Rev. Physiol. 51, 367-384.

[2] Brown, A.M. and Birnbaumer, L. (1990) Annu. Rev. Physiol. 52, 197-213.

[3] Hofmann, F., Flockerzi, V., Nastainczyk, W., Ruth, P. and Schneider, T. Current Topics cell. regul. (in press).

[4] Glossmann, H. and Striessnig, J. (1988) Vitamins Hormones 44, 154-327.

[5] Flockerzi, V., Oeken, J., Hofmann, F., Pelzer, D., Cavalie, A. and Trautwein, W. (1986) Nature 323, 66-68.

[6] Kannengiesser, U., Nalik, P. and Pongs, O. (1988) Proc. Natl. Acad. Sci. USA 85, 2969-2973.

[7] Tanabe, T., Beam, K.G., Powell, J.A. and Numa, S. (1988) Nature 336, 134-139.

[8] Tanabe, T., Mikami, A., Numa, S. and Beam, K.G. (1990) Nature 344, 451-453.

[9] Perez-Reyes, E., Kim, S.H., Lacerda, A.E., Horne, W., Wei, X., Rampe, D., Campbell, K.P., Brown, A.M. and Birnbaumer, L. (1989) Nature 340, 233-236.

[10] Ruth, P., Röhrkasten, R., Biel, M., Bosse, E., Regulla, S., Meyer, H.E., Flockerzi, V. and Hofmann, F. (1989) Science 245, 1115-1118.

[11] Bosse, E., Regulla, S., Biel, M., Ruth, P., Meyer, H.E., Flockerzi, V. and Hofmann, F. FEBS Lett. (in press).

[12] Tanabe, T., Takeshima, H., Mikami, A., Flockerzi, V., Takahashi, H., Kangawa, K., Kojima, M., Matsuo, H., Hirose, T. and Numa, S. (1987) Nature 328, 313-318.

[13] Ellis, S.B., Williams, M.E., Ways, N.R., Brenner, R., Sharp, A.H., Leung, A.T., Campbell, K.P., McKenna, E., Koch, W.J., Hui, A., Schwartz, A. and Harpold, M.M. (1988) Science $241,1661-1664$.

[14] Mikami, A., Imoto, K., Tanabe, T., Niidome, T., Mori, Y., Takeshima, H., Narumiya, S. and Numa, S. (1989) Nature 340, 230-233.

[15] Triggle, D.J., Langs, D.A. and Janis, R.A. (1989) Med. Res. Rev. 9, 123.

[16] Sambrook, J., Fritsch, E.F. and Maniatis, T. (1989) Molecular cloning: A Laboratory Manual, Cold Spring Harbor Laboratory, Cold Spring Harbor, NY.

[17] Methfessel, C., Witzemann, V., Takahashi, T., Mishina, M., Numa, S. and Sakmann, B. (1986) Pflügers Arch. 407, 577-588.

[18] Dascal, N., Snutch, T.P., Lübbert, H., Davidson, N. and Lester, H.A. (1986) Science 231, 1147-1150.

[19] Kameyama, M., Hofmann, F. and Trautwein, W. (1985) Pflügers Arch. 405, 285-293.

[20] Welling, C., Felbel, J., Peper, K. and Hofmann, F. (1990) Naunyn-Schmiedeberg's Arch. Pharmacol. 341, R62. 